\title{
Determinación del diámetro del cilindro central defectuoso en trozas podadas de pino radiata (Pinus radiata) mediante atenuación de radiación gamma
}

\author{
Determination of cylindrical defective core diameter in pruned \\ Pinus radiata (D. Don) logs using gamma radiation attenuation. \\ JOSE TOMAS KARSULOVIC C. ${ }^{1}$, MARIA INES DINATOR ${ }^{2}$, JOSE MORALES P. ${ }^{2}$ \\ VICTOR GAETE M. ${ }^{1}$, ANDRES BARRIOS R. ${ }^{1}$

\begin{abstract}
${ }^{1}$ Facultad de Ciencias Forestales, Universidad de Chile, Santa Rosa 11315, La Pintana, Santiago, Chile.
E-mail: tkarsulo@uchile.cl, vhgaete@uchile.cl.

${ }^{2}$ Facultad de Ciencias, Universidad de Chile. E-mail: mdinator@uchile.cl
\end{abstract}

\begin{abstract}
SUMMARY
This research, supported by FONDECYT Project \# 1990680, focused on the feasibility of the gamma ray attenuation method as a means of detecting the defective core size in commercial Pinus radiata (D. Don) pruned logs. The sample consisted of two $5 \mathrm{~m}$ commercial logs from the same tree which came from a 24 year old planting by Bosques Arauco S.A. The system arrayed in the Laboratory of Ionic Beams in the Faculty of Sciences of the University of Chile allowed the establishment of a precise geometry for the irradiation stage, reaching close to the preferred unit value for the build-up factor. In order to have precedence records for the effect of singularities (knots) in the attenuation, a simulation of the log using round plastic containers was carried out. This also allowed a comparison of results with the main experiment. An irradiation of the logs took place in three parallel planes at three different elevations with a collection time of 220 seconds per reading. The data on the attenuation was compared with the actual locations of defects plotted through progressive transverse sectioning of the logs. Subestimations determined the defect core size to be from 0.5 to $2.9 \mathrm{~cm}$ in diameter. The feasibility of this method to identify the knots in commercially shaped logs was demonstrated. Nevertheless, the accuracy of this method is not optimal, owing to the time required for each radiation. However, a reduced time-span may be possible by using stronger radioactive sources as well as thinner logs.
\end{abstract}

Keywords: gamma radiation, defect core size, radiata pine.

\section{RESUMEN}

Este estudio, desarrollado en el contexto del Proyecto FONDECYT 1990680, tuvo por finalidad analizar la factibilidad de la detección del Cilindro Central Defectuoso en trozas podadas de Pinus radiata (D. Don.), mediante atenuación de radiación gamma. El material de estudio consistió en dos trozas comerciales de una longitud aproximada de 5 metros de un mismo individuo de 24 años proveniente de una plantación de la empresa Bosques Arauco S.A. La puesta a punto del sistema espectroscópico instalado en el Laboratorio de Haces Iónicos de la Facultad de Ciencias de la Universidad de Chile, permitió establecer una geometría apropiada para la etapa de irradiación, lográndose un valor muy cercano a uno (valor ideal) para el factor de "Build - up". Con el objetivo de disponer de antecedentes previos del efecto de las singularidades (nudos) en la atenuación, se procedió a simular la troza con recipientes plásticos. Ello permitió, asimismo, apoyar el contraste de los resultados. Se procedió a irradiar con las trozas en tres planos paralelos a diferentes alturas, empleando un tiempo de colección de 220 segundos por lectura. Esta información expresada en atenuación del material y singularidades se contrastó con las posiciones reales del defecto (nudo) mediante la disección transversal y progresiva de las trozas, encontrándose, en general, subestimaciones del Cilindro Central Defectuoso en $0,5 \mathrm{~cm}$. como mínimo y 2,9 cm. como 
máximo. Se demostró la factibilidad del método para determinar el defecto nudo en trozas de tamaño comercial, aunque su grado de eficiencia no es el óptimo, producto de los tiempos empleados en la colección de los datos requeridos en cada irradiación. No obstante, la disminución en los tiempos de análisis, puede lograrse al emplear trozas de menor diámetro o adoptando fuentes radioactivas más intensas.

Palabras clave: radiación gamma, Cilindro Nudoso Central, pino radiata.

\section{INTRODUCCION}

En la economía chilena el sector forestal juega un importante papel en la generación de divisas, siendo los principales productos de exportación la celulosa y la madera aserrada proveniente principalmente del pino radiata. En la actualidad esta especie conforma el $98 \%$ del volumen total de las exportaciones de madera aserrada de Chile, correspondiendo a 1.140 millones de metros cúbicos en 1998 (1).

En los mercados externos existe una clara tendencia hacia el aumento de la demanda de madera libre de nudos de la especie pino radiata, por el mayor valor agregado que ello significa. En efecto, en Nueva Zelandia, el precio de las trozas podadas es cuatro veces superior a las no podadas. A fin de satisfacer estas exigencias por maderas de alta calidad, las principales empresas forestales del país han adoptado el desafío de maximizar la obtención de madera libre de nudos mediante la aplicación de tratamientos silviculturales como la poda.

La poda es una actividad que involucra la eliminación de ramas vivas o muertas del árbol, que permite la producción de madera libre de nudos, una vez que el crecimiento diametral del árbol cubre la zona de defectos, la cual queda así confinada a un cilindro central respecto al volumen total del fuste. De este modo, una adecuada programación de las podas permite controlar la dimensión de esta zona defectuosa, conocida como "Cilindro Central Defectuoso" (CCD).

El diámetro del CCD depende, entre otros factores, de la calidad del sitio y la oportunidad y número de podas que se ejecuten. La predicción de la cantidad de madera libre de nudos que se obtendrá al momento de la cosecha, en general se efectúa en base a estimaciones del CCD que se realizan mediante modelos de regresión. La alimentación de estos modelos debe provenir de mediciones directas del CCD en individuos obtenidos de muestreos estadísticos destructivos.
De lo anteriormente expuesto queda en evidencia la importancia de poder contar con metodologías que permitan establecer el diámetro del CCD sin necesidad de tener que destruir las muestras. Estas metodologías no destructivas presentan diversas ventajas tales como: apoyar los procesos de selección de trozas en el aserradero, experimentar con variadas condiciones sobre el material y realizar ensayos in situ de árboles en pie. Los grandes avances en tecnologías de escáner que, si bien hacen posible detectar los defectos internos con alta resolución (tomografía computarizada y resonancia magnética nuclear), no han sido suficientemente desarrollados para trabajar a velocidades de producción. La gran cantidad de proceso de datos produce problemas para su aplicación en tiempo real y no tiene, por ahora, posibilidad de ser aplicadas en el muestreo de árboles en pie.

Chang et al. (2) y Lindgren (3), utilizando los mismos equipos de uso médico demostraron aplicabilidad de la tomografía computarizada (CT) y la resonancia magnética nuclear (RMN) en el escaneo de trozas, detectando la presencia de nudos y otras singularidades internas. Benson et al. (4) ya señalaban bastante tiempo antes, la factibilidad del uso de la CT para proveer imágenes tridimensionales en la localización de defectos internos en trozas de pino radiata y en particular la zona interna defectuosa producida por la poda (CCD). Más recientemente Schad et al. (5) y Guddants (6) demuestran también la potencialidad que tienen los rayos $\mathrm{X}$ a través de la $\mathrm{CT}$ en el escaneo de trozas.

La radiación gamma se ha aplicado en la madera, centrándose preferentemente en el estudio de métodos densitométricos, pese a que algunos investigadores citan como alternativa el uso de radiación gamma para evitar algunos inconvenientes de los rayos $\mathrm{X}$ en la detección de defectos internos. Al respecto, cabe citar los trabajos realizados por los autores que demuestran la factibili- 
dad técnica de la aplicación del coeficiente de atenuación gamma para detectar nudos en piezas de madera aserrada de pino radiata (7) y la detección de pudrición central en trozas de lenga (8).

El propósito de este trabajo es avanzar hacia el desarrollo de un método basado en radiación gamma que discrimine en forma similar a los rayos X, el que, además de tener la ventaja de requerir sistemas más simples, hace factible su aplicación en la detección de defectos internos en árboles en pie a través del uso de equipos portátiles.

El CCD es la zona nudosa confinada al centro del fuste en la región podada del árbol para la obtención de madera libre de nudos. El CCD se conforma al cerrarse todos los nudos dejados por la poda de ramas, quedando los defectos confinados en una zona nudosa central. El CCD incluye, además, cualquier ensanchamiento debido a la sinuosidad del fuste al momento de la poda. En síntesis, los principales componentes que definen el diámetro del CCD, como se esquematiza en la figura 1, son: el diámetro sobre muñón de las ramas podadas (OSM), definido como el mayor diámetro medido sobre las ramas podadas al momento de la poda; el diámetro sobre oclusión (DSO), definido como el diámetro máximo sobre la oclusión del muñón (9), y la médula y la sinuosidad del fuste al momento de podar.

Emmingham y Fitzgerald (9) reportan diámetros del CCD cercanos a 10,16 cm. Lewis y Ferguson, citados por Palazuelos (11), señalan como apropiado unos $15 \mathrm{~cm}$. No obstante, para la realidad silvícola de Chile, un valor real puede estar entre $18 \mathrm{~cm}$ y $20 \mathrm{~cm}$ con podas realizadas a tiempo y con pocos problemas técnicos (11).

En la figura 1 se muestra la correspondencia existente entre los elementos principales que se relacionan con el CCD a nivel de un verticilo. El tamaño del CCD queda definido por el DSM más la variable profundidad de oclusión, que define el DSO, tal como se observa en la figura 1 .

Los nudos, generalmente presentan una densidad el doble que la de la madera sana, por lo que el haz de fotones debe sufrir mayor atenuación al atravesarlos, la que será tanto mayor mientras mayor sea su volumen ponderado en el recorrido

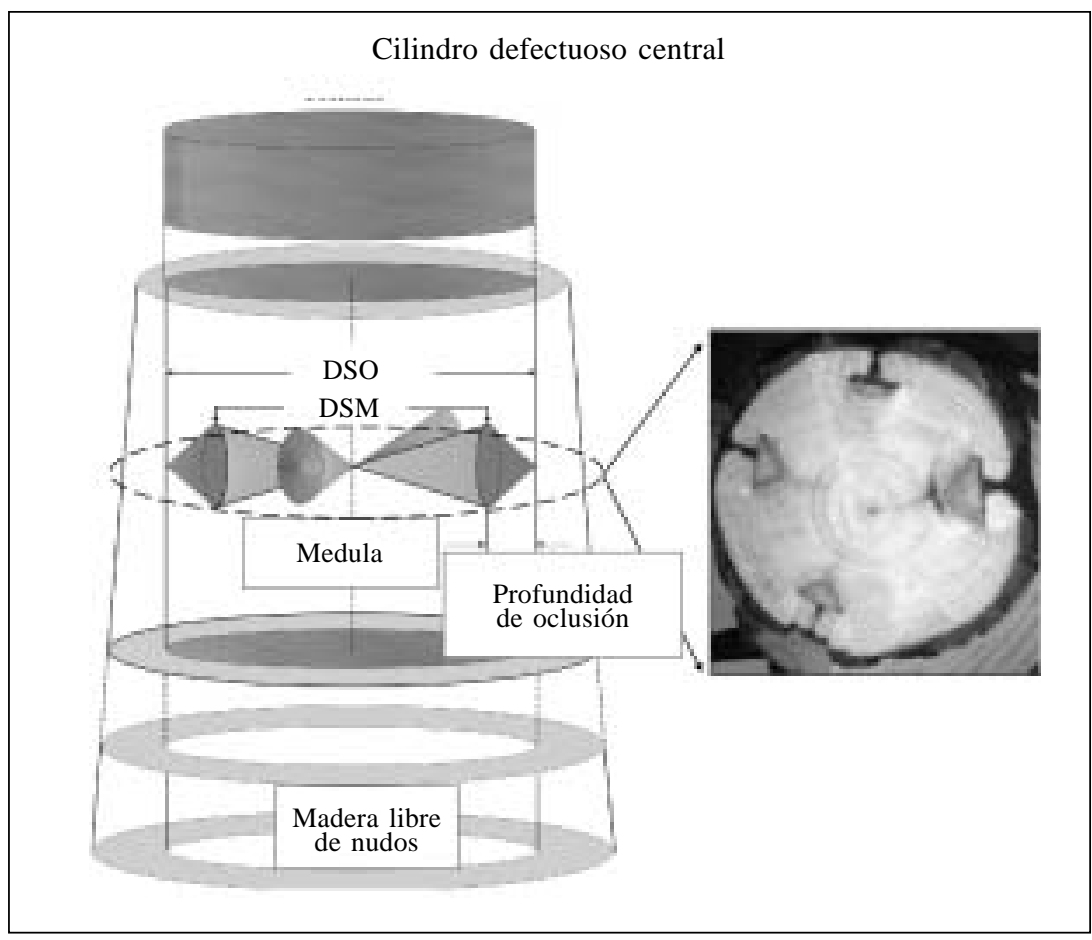

Figura 1: Esquema simplificado cilindro central defectuoso. Principales parámetros descriptores del CCD.

Cylindrical defective core, simplified scheme. Main description of CCD. 
del haz. Si es posible establecer las posiciones de los nudos más extremos efectuando una prospección en todos los verticilos que interesan en una troza o en una zona del fuste, será posible establecer las dimensiones del CCD.

Atenuación Gamma: Se refiere a la capacidad de un material para absorber en distintos grados una emisión de fotones de una intensidad dada (Io). La ocurrencia de interacción de los fotones con la materia obedece a una probabilidad conjunta. Dicha probabilidad, llamada coeficiente de atenuación lineal $\left(\mu_{L}\right)$, la que está formada por la suma de las probabilidades asociadas a tres tipos de interacciones: efecto fotoeléctrico, efecto Compton y formación de pares (Knoll (12).

En relación a la cantidad de fotones incidentes (Io), emergentes (I) y el espesor del material en cuestión $(\mathrm{t})$, se relacionan con el coeficiente de atenuación lineal $\mu_{\mathrm{L}}$ de acuerdo a una relación exponencial, llamada ecuación de Beer-Lambert:

$$
\frac{I}{I o}=e^{-\mu_{L} \cdot t}
$$

El coeficiente de atenuación lineal está relacionado con el coeficiente de atenuación másico $\mu_{1}$ a través de la siguiente relación:

$$
\mu_{1}=\mu_{\rho} \rho
$$

Donde 1 corresponde a la densidad del material atenuado.

El coeficiente de atenuación másico depende de los elementos químicos fundamentales que conforman el material, por lo tanto en un material dado, el coeficiente de atenuación másico corresponde al valor ponderado $\left(\mathrm{w}_{\mathrm{i}}\right)$ de los coeficientes de cada componente $\left(\mu_{\mathrm{p}}\right)_{\mathrm{i}}$, luego $\mathrm{m}_{\mathrm{r}}$ se expresa como:

$$
\mu_{\rho}=\sum_{1}^{n} w_{i}(\mu \rho)_{i}
$$

No obstante que cabría esperar que el conteo de fotones corresponda a aquellos que no fueron absorbidos, lo cual sucedería con una "buena geometría" (12). En la práctica ello no suele ocurrir, pues de la totalidad de fotones que llegan al detector, existe otra clase de fotones también contabilizados que corresponden a productos de interacciones (fundamentalmente efecto Compton y producción de pares) con el material absorbedor o con el mismo blindaje. Ello hace necesaria una corrección en la relación de atenuación gamma, llamada "Build-up Factor" (B), cuyo valor depende de la energía del fotón, del espesor del material atenuador y de la geometría del sistema.

Así la ecuación [1], adopta la siguiente forma:

$$
\frac{I}{I o}=B(t, h v) \cdot e^{-\mu \cdot t}
$$

Donde: $\mathrm{t}=$ espesor material atenuador; $\mathrm{h} v=$ energía de los fotones

\section{MATERIAL Y METODOS}

Se obtuvo material de dos trozas provenientes de un individuo proporcionado por Bosques Arauco S.A. El material se originó en un predio de 274 há ubicado en Curanilahue, plantado en 1976, con podas a los 9 y 11 años. El rodal presentaba altura dominante de 34 metros y DAP promedio de $41,2 \mathrm{~cm}$. De este individuo, se procedió a seleccionar dos trozas de aproximadamente $70 \mathrm{~cm}$ de longitud cada una, con la precaución de que dicho material incluyera un área nudosa y una buena proporción de madera libre de nudos. Los diámetros de las trozas fueron de $36 \mathrm{~cm}$ aproximadamente.

Las trozas obtenidas fueron pintadas en ambas caras con pintura de aluminio, para luego recibir la aplicación de una película plástica. Ello con el objeto de evitar la pérdida de humedad de las muestras en la manipulación previa a los ensayos de laboratorio.

Posteriormente se efectuaron despuntes de 5 $\mathrm{cm}$ en ambas trozas, con el objeto de eliminar el material afectado por desecamiento. Luego, las trozas volvieron a sellarse para mantener sus condiciones de humedad en las etapas sucesivas del estudio.

En laboratorio se definió un sistema de referencia para realizar las irradiaciones sobre las trozas consistente en un sistema de ejes coordenados (XYZ), el que fue concebido de tal manera que su origen coincidiera con el centro del plato portamuestras del banco de pruebas. (figura 2).

La caracterización física del material se llevó a cabo a partir de dos rodelas obtenidas de cada troza, de las cuales se obtuvieron probetas de $5 \times 5 \times 4 \mathrm{~cm}$, registrándose la posición de las probetas mediante un sistema de ejes coordenados en el plano de la rodela. 


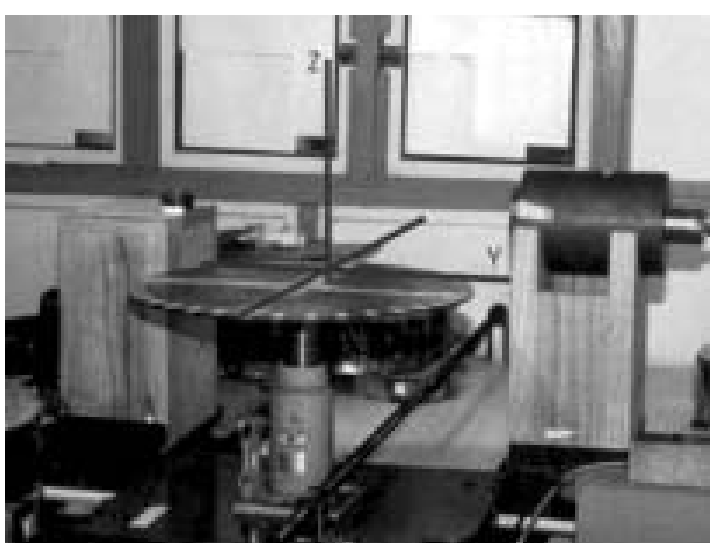

Figura 2: Sistema de referencia de tres coordenadas rectangulares (Banco colimación amplia, Facultad de Ciencias - U. de Chile).

Referential three-dimensional coordinate system (wide collimation bench, Faculty of Sciences, University of Chile).
Se confeccionaron perfiles bidimensionales del contenido de humedad $(\mathrm{CH} \%)$ y densidades (a contenido de humedad de ensayo, básica y anhidra), con el objetivo de disponer de los antecedentes necesarios para el análisis de la atenuación. El diagrama de extracción de probetas se muestra en la figura 3 .

Bancos de ensayos: Se utilizaron dos bancos de irradiación, el primero (figura 4) con un haz colimado (colimación fina $/ 3 \mathrm{~mm}$ ), para la determinación del coeficiente de atenuación másico, y el segundo, con colimación amplia, figura 2 , se utilizó para la irradiación de las trozas. Cabe hacer notar que este banco está diseñado de modo que permitiese desplazamientos con tres grados de libertad.

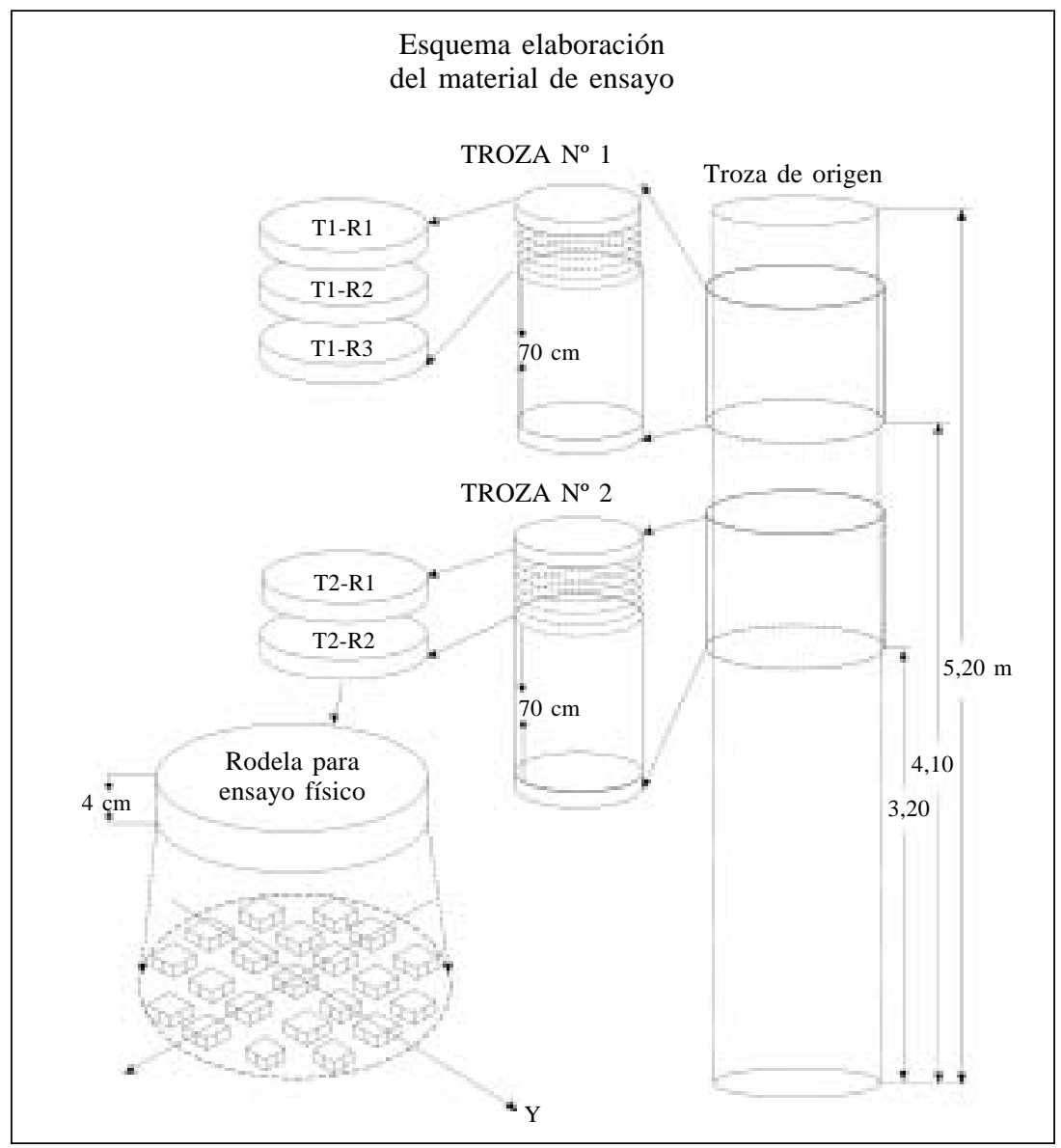

Figura 3: Obtención del material para su caracterización física.

Sample extraction diagram for physical characterization. 


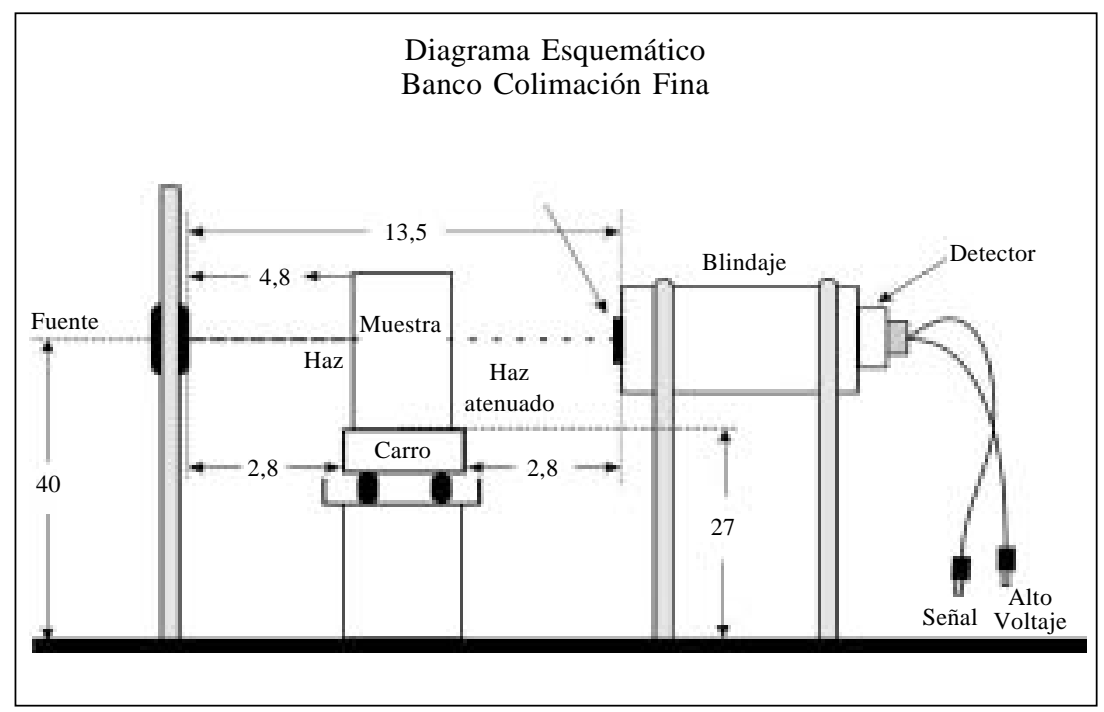

Figura 4: Esquema Banco Colimación Fina. (Las distancias se indican en centímetros). Narrow collimation bench scheme (distances in centimeters).

\section{CALIBRACIÓN SISTEMA ESPECTROSCÓPICO GAMMA}

Alineación angular de la fuente: Considerando que la emisión del haz asemeja a un volumen cónico (figura 5), se procedió a centrar su dirección horizontal hacia el detector y definir áreas de seguridad, precisando de esta manera la abertura máxima de dicho cono imaginario. La posición angular $0^{\circ}$ se estableció como el punto con máximo registro de fotones. Se registraron lecturas no atenuadas (Io), con un tiempo de colección de 30 segundos para un paso angular de 5 grados.

Relación entre "distancia fuente" e intensidad

Sobre el mismo banco de pruebas se efectuó una verificación de la relación entre la distancia "fuente - detector" (r) y la intensidad (Io). Dicha intensidad o cantidad de cuentas no atenuadas, fue obtenida para 30 segundos de colección. Luego se realizó el análisis de los datos, en base a una comparación con la relación teórica indicada a continuación, que debe cumplirse para $r$ e Io:

$$
I o=a \cdot \frac{1}{r^{2}}
$$

En donde el cálculo de la constante " $a$ ", se obtuvo con el valor $\mathrm{r}$ máximo $(85,65 \mathrm{~cm})$ y la intensidad "Io" asociada. Luego se obtuvo una curva teórica, en función de r; la cual se comparó con la curva experimental.

Finalmente se establecieron dos ajustes (potencial y lineal), comparando la tendencia teórica con la experimental. El análisis completo se llevó a cabo para las dos posiciones verticales extremas del soporte de muestras: 5 y 15 centímetros bajo el haz (posición máxima y mínima respectivamente).

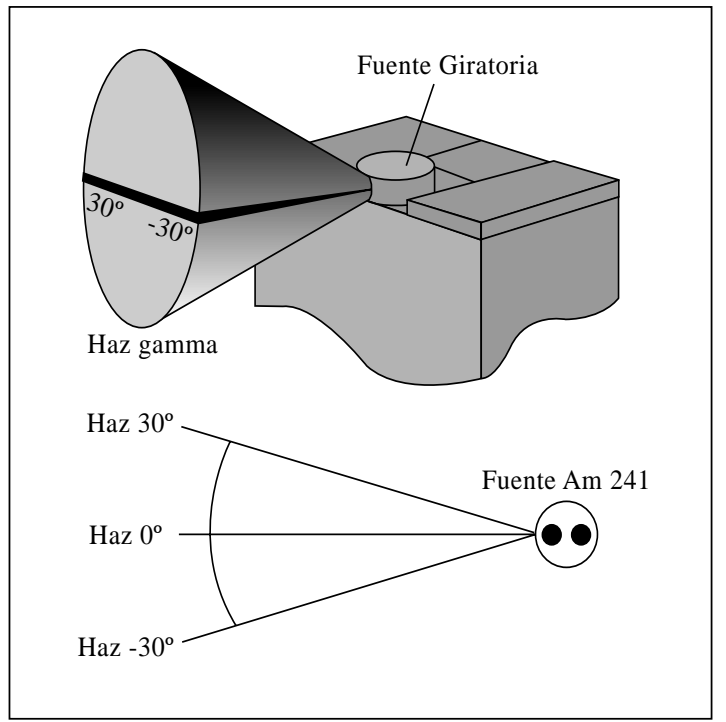

Figura 5: Alineación angular fuente Am 241. Angular alignment of Am 241 source. 
Efecto de la geometría de las muestras: El estudio del efecto de la geometría cilíndrica de las muestras sobre la espectroscopia se llevó a cabo mediante una simulación, utilizando recipientes de plástico, con diámetros semejantes a las de las trozas empleadas. Se analizó el comportamiento de la atenuación con los recipientes conteniendo agua. Antes de irradiar se determinó el tiempo apropiado de colección para un error estadístico no mayor a $1 \%$ (12). Para evaluar la calidad de la geometría del banco de pruebas, se realizó la comparación de los diámetros reales de los bidones con los diámetros estimados según las lecturas de las atenuaciones del haz de radiación gamma.

Determinación del Coeficiente de Atenuación Másico $\mu_{p}$ : Se determinó el coeficiente de atenuación másico $\left(\mu_{\mathrm{p}}\right)$ en madera de pino radiata utilizando el banco de colimación fina, considerando dos variables: contenido de humedad (estado verde, estado ambiente y estado anhidro) y la posición diametral del material irradiado en la rodela. Ambas variables se sometieron a un análisis conjunto (su efecto combinado sobre $\mu_{\mathrm{r}}$ ). El coeficiente de atenuación másico $\left(\mu_{\mathrm{r}}\right)$ se determinó a partir de la ecuación [1], procediéndose a efectuar un análisis de la significancia estadística de estos factores sobre $\mu_{\mathrm{p}}$.

Irradiación de Simulación de Trozas con Defectos. El ensayo de simulación consistió en reproducir, lo más fielmente posible, las características de las trozas reales (incluyendo el CCD); en particular las condiciones de densidad de los materiales atenuadores y la posición espacial de los defectos. Dentro de los recipientes utilizados para estudiar el efecto de la geometría de las muestras, se insertó un vástago de PVC, lleno con agua, con dimensiones, geometría y posición similar a un verticilo real (figura 6).

Luego los recipientes fueron llenados con aceite quemado de motor, cuya densidad gravimétrica promedio fue $0,86 \mathrm{~g} / \mathrm{cm}^{3}$, la cual se asemeja a la densidad promedio en madera de pino radiata en condiciones verdes, ello con el objeto de reproducir la relación de densidades promedio entre madera húmeda y nudos. Las irradiaciones se efectuaron en dos direcciones ortogonales, coincidentes con las posiciones de los nudos. Posteriormente se retiró el verticilo simulado, efectuándose irra-
Configuración defecto simulado
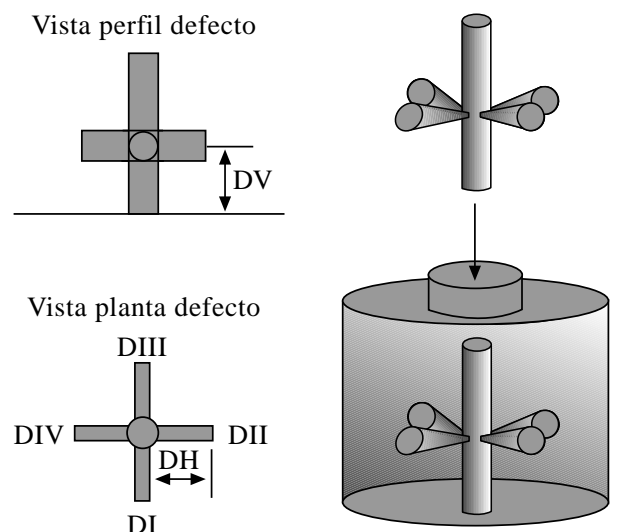

DI-DIV. Defectos simulados (nudos)

DH: Proyección horizontal defecto $(\mathrm{cm})$

DV: Proyección vertical defecto $(\mathrm{cm})$

Figura 6: Esquematización defecto simulado.

Simulated defect scheme.

diaciones según cuerdas paralelas con el objeto de comparar los perfiles de atenuación en las dos condiciones.

Análisis del Factor de Build-up. Con los datos de atenuación obtenidos en los ensayos descritos, se procedió a calcular el factor de Build-Up para el banco de colimación amplia, utilizando los recipientes llenos de aceite con y sin la inclusión del defecto simulado. Luego se determinó dicho factor según el espesor " $t$ " del material. Knoll (12), indica que el valor del factor debe tender a la unidad cuando " $t$ " se hace cero, según la siguiente relación:

$$
\ln [I]=M \cdot t+N
$$

Donde:

$$
\begin{aligned}
& M=\mu_{\rho} \cdot \rho \\
& N=\ln [l o]+\ln [B(t)]
\end{aligned}
$$

Luego, a partir de los datos de atenuación, se realizó un ajuste lineal de la $\ln [I]=M \cdot t+N$ para cada caso.

Irradiación de Trozas. La definición del paso horizontal $(\mathrm{PH})$ se prefijó en $1 \mathrm{~cm}$ para todo el pro- 
ceso de irradiación. El número y la altura de los planos horizontales de irradiación se definieron en función de la posición vertical de las marcas de nudo en la corteza, teniendo en consideración la extensión vertical de las mismas. En ambas trozas se utilizó un tiempo de colecta de 220 segundos, con el objeto de obtener un error estadístico aproximado de $1 \%$, asociado al área del peak fotoeléctrico.

El análisis se basó fundamentalmente en la geometría cilíndrica de la troza, en donde los perfiles de intensidades atenuadas se contrastaron con la geometría de un perfil sin defectos, de acuerdo a los resultados de simulación, para visualizar irregularidades atribuibles al defecto nudo.

\section{RESULTADOS Y DISCUSION}

Caracterización física-Contenido de humedad: En el cuadro 1 se presentan valores de contenidos de humedad en porcentaje $(\mathrm{CH} \%)$ para el material en estudio (trozas 1 y 2).

Entre todos los valores de contenidos de humedad mínimos, el menor fue de 43,3\%, ubicándose todos ellos en las zonas central y periférica de cada rodela. En cambio, los valores de contenidos máximos de humedad, ubicados de prefe-

\section{CUADRO 1}

Contenidos de humedad de las trozas irradiadas. Moisture content of irradiated $\log$ s.

\begin{tabular}{|lrr|}
\hline Contenidos de & Troza 1 & Troza 2 \\
Humedad (\%) & & \\
\hline Mínimo & 46,95 & 43,30 \\
Máximo & 171,04 & 184,33 \\
Promedio & 135,45 & 141,10 \\
Desviación estándar & 31,61 & 44,52 \\
\hline
\end{tabular}

rencia en la zona sub-periférica, arrojaron un máximo de 184,3\% (figura 7).

\section{DENSIDADES}

A modo de ejemplo, en la figura 8 se muestra un perfil de distribución de densidades de una de las trozas ensayadas (troza 1), indicando además la posición de las probetas en la rodela mediante ejes coordenados graduados en $\mathrm{cm}$. Se compararon los distintos perfiles de densidad con el contenido de humedad de ensayo, anhidra y básica. Es importante esta comparación, por cuanto la atenuación de radiación gamma se aprecia correlacionada muy cercanamente con el contenido de humedad.

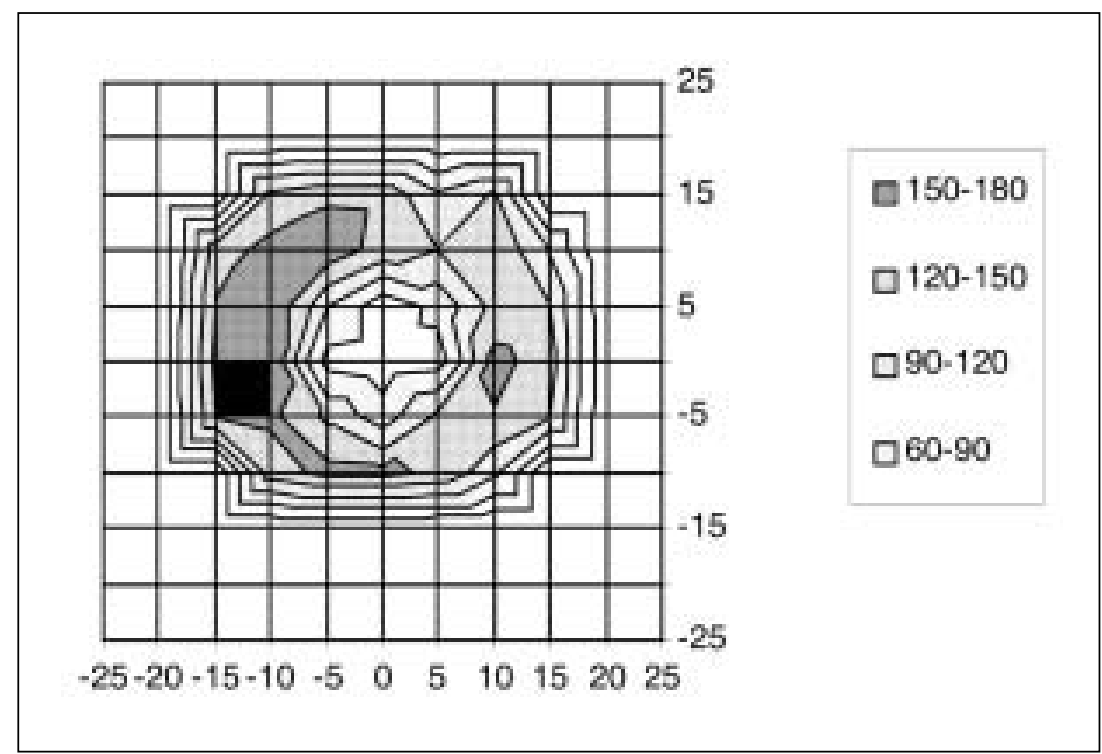

Figura 7. Contenidos de humedad de la troza $2(\%)$.

Moisture content of $\log$ number $2(\%)$. 


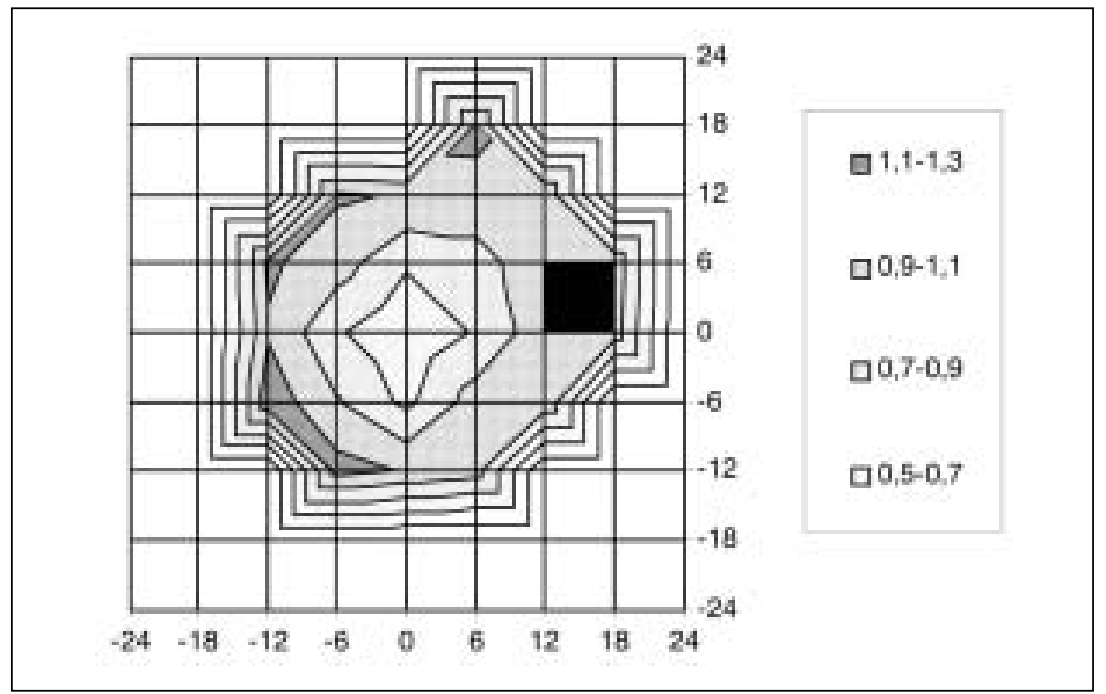

Figura 8: Distribución de densidad a $\mathrm{CH}$ ensayo.

Density profile and assay moisture content.

\section{CALIBRACION SISTEMA ESPECTROSCOPICO GAMMA}

Alineación angular de la fuente: La abertura efectiva del cono que representa la emisión de fotones gamma, se determinó en $20^{\circ}$ o $\left[-10^{\circ} \sim+10^{\circ}\right]$ aproximadamente, si se consideran valores a partir de 10.000 cuentas como mínimo. Dicha información es importante para determinar dos puntos significativos: en primer lugar la seguridad del operador al evitar la exposición accidental a la radiación, y en segundo lugar permite determinar el rango efectivo de irradiación horizontal de cualquier muestra. Este último hecho permite conocer mejor las posiciones afectadas por efecto de borde (figura 9).

Verificación Relación Distancia "Fuente-Detector" e Intensidad: Utilizando la relación dada por ecuación [5], los valores del parámetro " $a$ " (utilizado en la construcción de las curvas teóricas $r$ v/s Io) son los siguientes:

$a=2,86 \times 10^{9}$ (posición mínima del soporte de muestras - $15 \mathrm{~cm}$ bajo haz)

$a=2,87 \times 10^{9}$ (posición máxima del soporte de muestras $-5 \mathrm{~cm}$ bajo haz)

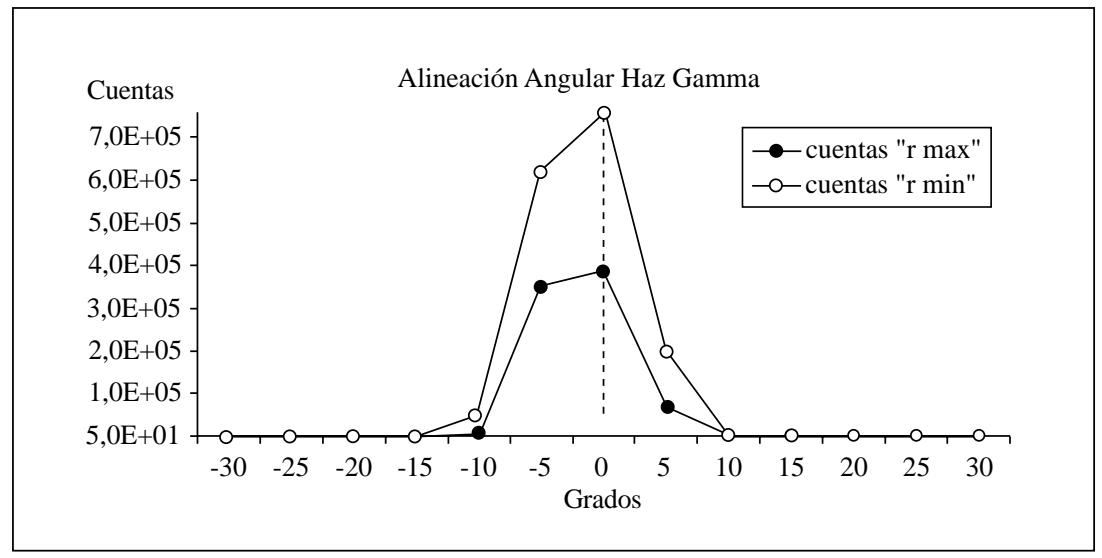

Figura 9. Intensidad no atenuada, para distintas posiciones angulares de la fuente. Non-attenuated intensity, for different angular positions of the source. 
De acuerdo al ajuste potencial llevado a cabo, resultaron las relaciones funcionales, con los respectivos valores de los coeficientes (cuadro 2):

Ajuste potencial: $\quad Y=\alpha \cdot X^{B}$

\section{CUADRO 2}

Resultados del ajuste de regresión. Regression adjusted results.

\begin{tabular}{|l|c|c|c|c|}
\hline Modelo & Posic. soporte & $\alpha$ & $\beta$ & $\mathrm{R}^{2}$ \\
\hline Potencial & Mínima & 6,12 E8 & $-1,650$ & 0,998 \\
& Máxima & 6,14 E8 & $-1,651$ & 0,998 \\
\hline
\end{tabular}

El coeficiente de determinación correspondiente al ajuste potencial está en pleno acuerdo con la relación teórica dada por la ecuación [5]. Se establece que no hay un efecto importante del soporte de las muestras, según su posición vertical, sobre el conteo de fotones por parte del detector (scattering).

Análisis del efecto de la geometría de las muestras de modelación (Atenuación): Se determinaron los valores de $\mathrm{I}_{0}$ de modo de que el error estadístico asociado en la lectura no fuese superior al $1 \%$, variando entre $7,7 \times 10^{4}$ y $15,2 \times 10^{4}$.

A modo de ejemplo, en la figura 10 se muestran las curvas de atenuación para uno de los recipientes de modelación, semejante en diámetro a la troza 2, vacío y conteniendo agua. En esta figura se observa una buena sensibilidad del método en la reproducción de la geometría del contenedor en ambos casos, no superando el 4,2\% de error.

Determinación del Factor "Build up-B(t)". En relación con los datos de atenuación obtenidos en la etapa de irradiación de cilindros con aceite, y los valores respectivos de espesores de material; se entregan, a modo de ejemplo, los resultados para el mismo recipiente mencionado anteriormente. En éste se efectuaron dos recorridos o planos horizontales de irradiación. Derivándose dos regresiones lineales con alto grado de correlación $\left(\mathrm{R}_{1}^{2}=0,9949\right.$ y $\left.\mathrm{R}_{2}{ }_{2}=0,9939\right)$. Se obtuvieron valores de $\mathrm{B}(\mathrm{t})$ con una diferencia de $11 \%$ y $14 \%$ para los planos de irradiación 1 y 2 respectivamente $[\mathrm{B}(\mathrm{t})=1,11$ para plano $1 \mathrm{y}$ $\mathrm{B}(\mathrm{t})=1,14$ para plano 2$]$.

Determinación del coeficiente de atenuación másico: Se determinó que para no superar un error muestral del 5\% de debió tomar un número mínimo de 10 lecturas. De los datos tomados de 10 bloques provenientes de la Troza 2 se realizó una ANDEVA (prueba F), a fin de observar los efectos de la posición diametral y del contenido de humedad, sobre el valor de $\mu_{\mathrm{p}}$. En cada bloque de la vigueta 1 (6 bloques) se tomaron siete observaciones, y para la vigueta 2 (4 bloques), seis observaciones (cuadros 3 y 4 ).

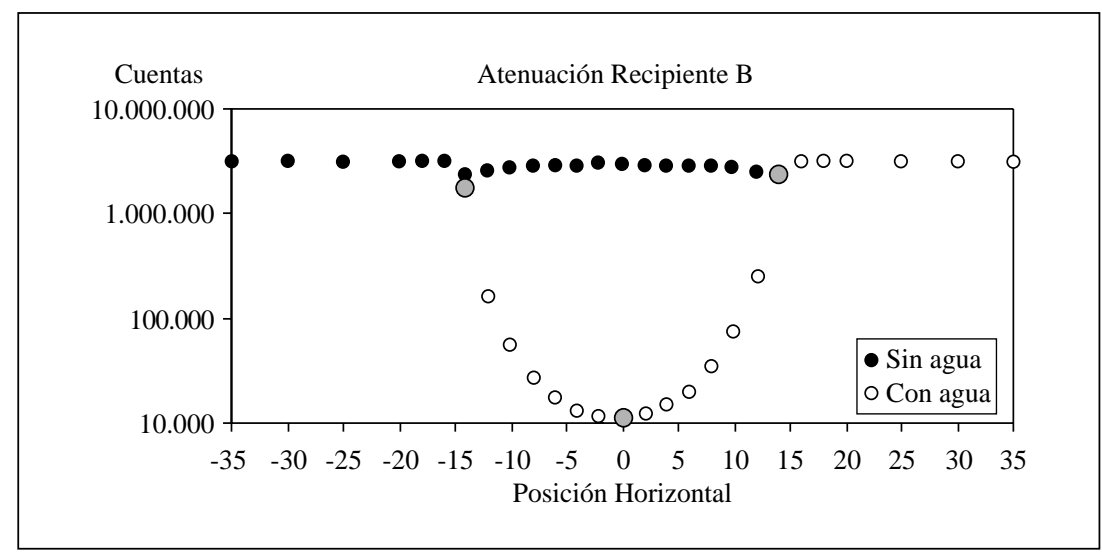

Figura 10. Análisis de la geometría de las muestras.

Sample geometry analysis. 


\section{CUADRO 3}

Coeficientes de atenuación másico promedio en vigueta 1 .

Average masic attenuation coefficient in beam number 1 .

\begin{tabular}{|l|c|c|c|}
\hline & Húmedo & Ambiente & Anhidro \\
\hline & $\mu_{\mathrm{p}}\left(\mathrm{cm}^{2} / \mathrm{g}\right)$ & $\mu_{\mathrm{p}}\left(\mathrm{cm}^{2} / \mathrm{g}\right)$ & $\mu_{\mathrm{p}}\left(\mathrm{cm}^{2} / \mathrm{g}\right)$ \\
\hline Promedio & 0,192 & 0,187 & 0,188 \\
Desv. Est. & 0,014 & 0,006 & 0,002 \\
Coef. Variac. & 7,1 & 3,2 & 1,0 \\
\hline
\end{tabular}

\section{CUADRO 4}

Coeficientes de atenuación másico promedio en vigueta 2 .

Average masic attenuation coefficient in beam number 2 .

\begin{tabular}{|l|c|c|c|}
\hline & Húmedo & Ambiente & Anhidro \\
\hline & $\mu_{\mathrm{p}}\left(\mathrm{cm}^{2} / \mathrm{g}\right)$ & $\mu_{\mathrm{p}}\left(\mathrm{cm}^{2} / \mathrm{g}\right)$ & $\mu_{\mathrm{p}}\left(\mathrm{cm}^{2} / \mathrm{g}\right)$ \\
\hline Promedio & 0,198 & 0,188 & 0,188 \\
Des. Estándar & 0,005 & 0,002 & 0,005 \\
Coef. Variac. & 2,5 & 1,0 & 2,8 \\
\hline
\end{tabular}

Los valores de $\mu_{\mathrm{p}}$ muestran un comportamiento esperado en función a los contenidos de humedad. Ello porque en condiciones de menor humedad, el material irradiado debería mostrar una leve diferencia en su capacidad de atenuar (menor valor de $\left.\mu_{\mathrm{p}}\right)$. Además los valores indicados para las condiciones de humedad en ensayo y anhidro, son coherentes según estudios precedentes reportados por Karsulovic et. al. (7), en donde se indica un valor de 0,192 $\pm 0,002$ para un contenido de humedad de un $10 \%$. Los resultados de la prueba de ANDEVA para ambas viguetas, coinciden en identificar los efectos del factor contenido de humedad como relevante con un $95 \%$ de confianza. En el caso del factor posición diametral, se determinó que no hay efectos significativos al mismo nivel de confianza.

Irradiación de simulación de trozas con defectos: Con los resultados de irradiación en dos direcciones ortogonales se construyeron perfiles de atenuación, para recipientes con aceite. En la figura 11 se presenta el perfil de una trayectoria de irradiación, siendo su correspondiente ortogonal muy similar.

Se aprecian las diferencias de atenuación, al comparar los perfiles con y sin defecto. La diferencia entre las dimensiones, en proyección horizontal, reales y estimadas de los defectos alcanza un $10 \%$ para ambas trayectorias.

Resultados irradiación de trozas: La presencia de nudos en los verticilos, provoca una alteración en la forma parabólica del perfil de atenuación. Esto último puede observarse en los siguientes gráficos, mostrándose las alteraciones propias debidas a la irregularidad de la geometría de la troza. En las figuras 13 y 14 se presentan perfiles de atenuación para el plano medio de la zona de verticilo de la troza 2 (figura 14).

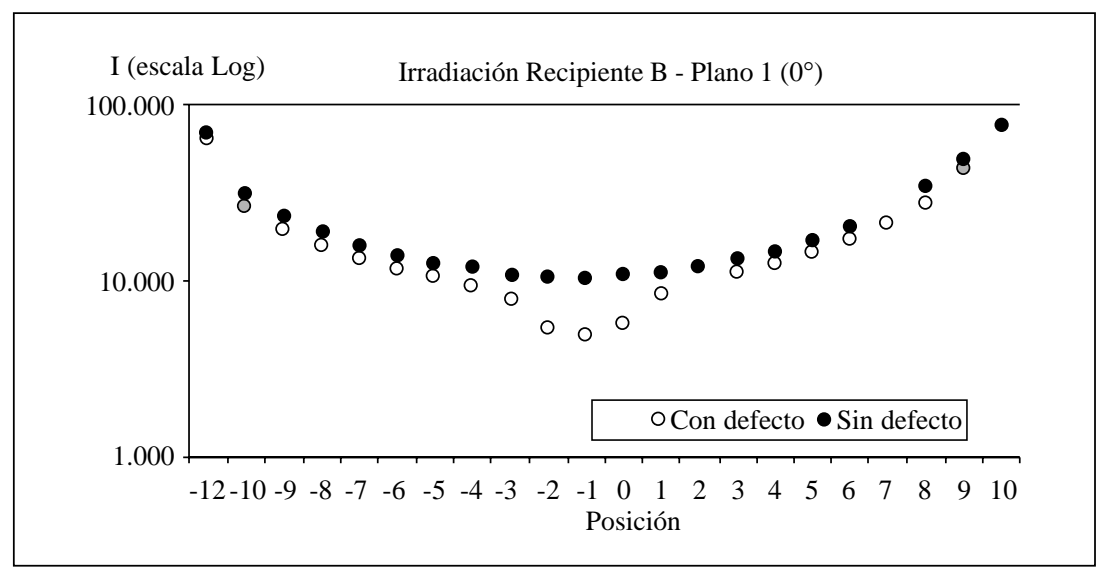

Figura 11. Atenuación recipiente con aceite (trayectoria 1).

Oil container attenuation (path number 1). 
Según el comportamiento de las atenuaciones, el comienzo de irregularidad en la tendencia de cada perfil denota la presencia de nudo. Ello se estableció de acuerdo al perfil esperado (de forma homogénea) en una troza sin defectos, en base a los resultados de simulación de trozas sin defectos. Existen, sin embargo, casos, en donde no se logra apreciar dicha irregularidad o alteración en la tendencia del perfil, por lo que no se puede asegurar la presencia de defecto.

El desarrollo horizontal del defecto en centímetros (dimensiones predichas) se comparó con las extensiones horizontales reales del defecto (cuadro 5). La extensión real del defecto se obtuvo con la medida de la distancia de los extremos derecho e izquierdo de la proyección de los nudos sobre los ejes coordenados " $\mathrm{X}$ " e "Y" ya descritos. Así, los porcentajes de variación entre la predicción del defecto y la situación real, se realizó según: (valor predicho - valor real)/(valor real) x 100 .

En función al inicio y término de la irregularidad (predichos) en los perfiles de atenuación registradas en el cuadro 5 se obtienen igual cantidad de sobreestimaciones y subestimaciones para la longitud horizontal total del defecto, alcanzando un valor constante aproximado de $3 \%$ para la orientación $0^{\circ}$ del haz. En cambio, para la orientación $90^{\circ}$, ocurren subestimaciones de las longitudes horizontales con un máximo de $8,57 \%$ y un mínimo de 2,8\% (sobreestimación).

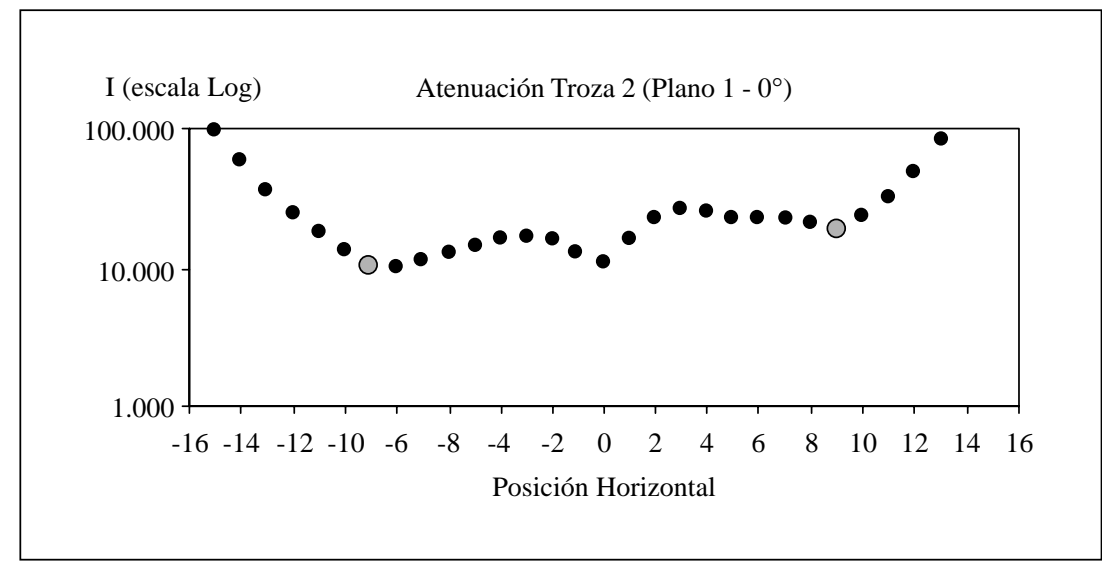

Figura 12. Atenuación Troza 2 - Ángulo $0^{\circ}(8 \mathrm{~cm}$. Sobre el soporte de muestras). $\log 2$ attenuation $-0^{\circ}$ angle $(8 \mathrm{~cm}$ above the sample carrier).

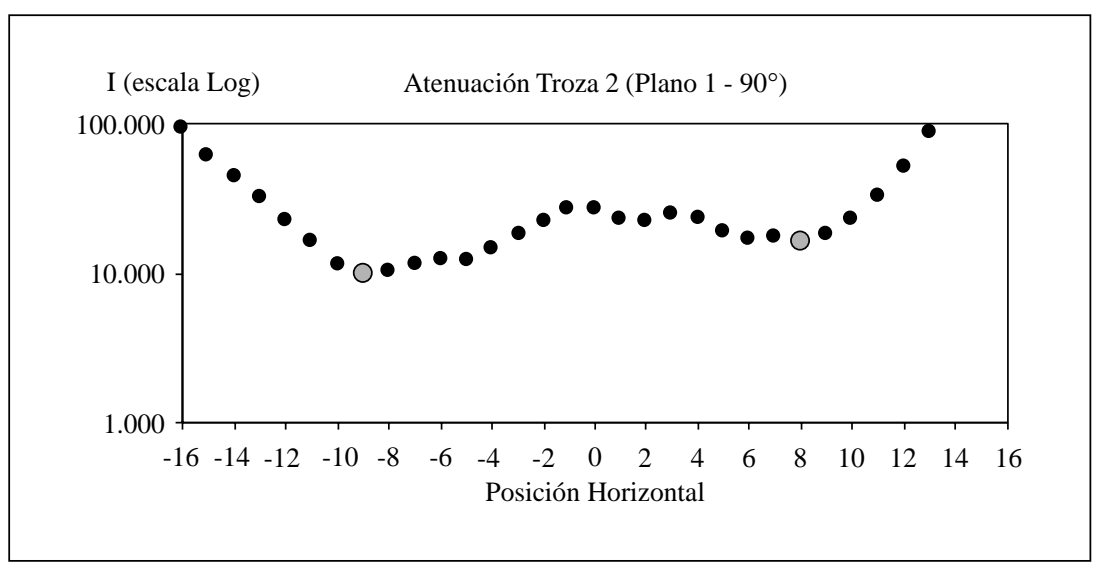

Figura 13. Atenuación Troza 2 - Ángulo $90^{\circ}$ (8 cm sobre el soporte de muestras). Log 2 attenuation $-90^{\circ}$ angle ( $8 \mathrm{~cm}$ above the sample carrier). 


\section{CUADRO 5}

Desarrollo horizontal del defecto en centímetros (dimensiones predichas). Horizontal defect display in centimeters (predicted dimensions).

\begin{tabular}{|c|c|c|c|c|c|c|}
\hline Plano & Ángulo & Extremo Izq. & Extremo Der. & Total & Diferencia & $\%$ \\
\hline \multirow{2}{*}{2} & $0^{\circ}$ & 8 & 9 & 17 & 0,5 & 3,00 \\
& $90^{\circ}$ & 9 & 8 & 17 & $-0,5$ & $-2,80$ \\
& $0^{\circ}$ & 8 & 9 & 17 & 0,5 & 3,00 \\
& $90^{\circ}$ & 8 & 8 & 16 & $-0,5$ & $-8,57$ \\
& $0^{\circ}$ & 8 & 8 & 16 & $-0,5$ & $-3,00$ \\
& $90^{\circ}$ & 9 & 8 & 17 & 0,5 & 2,80 \\
\hline
\end{tabular}

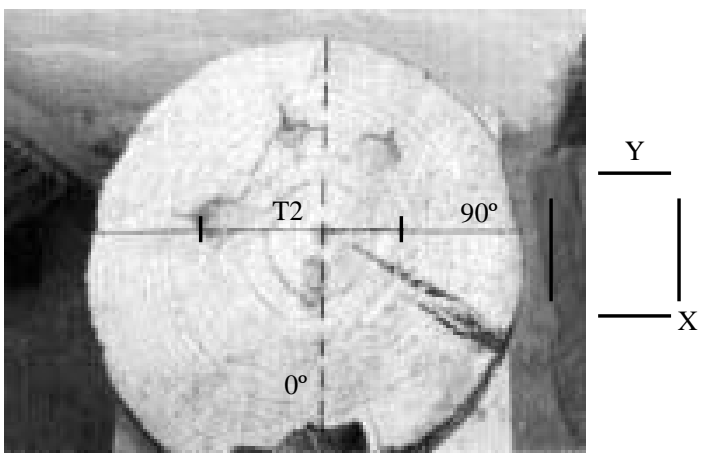

Figura 14. Proyecciones en Troza 2 (Eje $\mathrm{X}=$ orientación $0^{\circ}$ - Eje $\mathrm{Y}=$ orientación $\left.90^{\circ}\right)$.

Projections on log number $2\left(\mathrm{X}\right.$ axis $=0^{\circ}$ orientation, $\mathrm{Y}$ axis $=90^{\circ}$ orientation) .

Con respecto a las sobreestimaciones, y cómo se determinó en estudios precedentes (12), ello se debería a la mayor densidad de la madera en torno a los nudos, lo que hace parecer al nudo, de mayor dimensión de lo real. Por el contrario, las subestimaciones pueden explicarse por el bajo peso ponderado del material nudoso en relación al total de material atravesado por el haz, es decir, una baja relación "material nudo/madera sana", lo que disminuye la sensibilidad de detección del nudo.

\section{CONCLUSIONES}

El contenido de humedad de la madera, afecta significativamente al coeficiente de atenuación másico, en promedio $5 \%$ de variación, para un nivel de confianza $95 \%$.
La metodología utilizada permitió evidenciar el tamaño del CCD en un rango de precisión de un $4 \%$ a $12 \%$, correspondiente a valores de los diámetros promedio del CCD de $16,5 \mathrm{~cm}$ para la troza 1 y $16,6 \mathrm{~cm}$ para la troza 2 , con una subestimación máxima de $15,34 \%$ y $8,57 \%$ respectivamente.

El tiempo empleado en la toma de los datos y generación de resultados es muy extenso para ser implementado a escala industrial. El problema puede solucionarse con el diseño de equipos mas eficientes (bancos de pruebas con mayor número de detectores o fuentes radiactivas más intensas, etc.).

\section{AGRADECIMIENTOS}

Los autores agradecen a FONDECYT por el financiamiento para la realización de este estudio y a Bosques Arauco S.A. por el aporte de material.

\section{BIBLIOGRAFIA}

(1) INFOR. Resúmenes estadísticas forestales. 2002. EN: www.infor.cl.

(2) CHANG, S.J., J.R. OLSON, P. C. WANG. NMR Images of Internal Features in Wood. Forest Products Journal, 1989, vol. 39, $\mathrm{N}^{\circ}$ 6, p. 43-49.

(3) LINDGREN, L.O. Medical CAT - Scanning: X-Ray absortion coefficients, CT-Numbers and their relation to wood density. Wood Sci. Technol., 1991, vol. 25. p. 341-349.

(4) BENSON D.M., K.L. KNOWLES, F.I. THOMPSON, D.T. CROWN. Computed tomographie scanning for the detection of defects within logs. Forest Research 'Institute New Zealand - Forest Service., 1982, Fr. Bulletin $\mathrm{N}^{\circ} 8$, p. 9.

(5) SCHAD, K. et al. Nondestructive methods for detecting defects in softwood logs. Forest Products Laboratory, Research Paper FPL-RP, 1996, vol. 546. p. 13. 
(6) GUDDANTS, S. Replicating sawmill sawing with topsan using C.T. images of a full-Iength hardwood log. Forest Products Journal, 1998, vol 48, № 1, p. 72-75.

(7) KARSULOVIC, J. et al. The use of linear attenuation coefficients of gamma radiation for detecting knots in Pinus radiata. Forest Products Journal, 1999, vol. 49, No 2, p. 73-76.

(8) KARSUlOVIC, J.T., M.I. DINATOR, R. MORALES. Detección de pudrición central en trozas de Lenga ( $\mathrm{No}$ thofagus pumilio (Poepp. Et Endl] Krasser) mediante atenuación de Radiación Gamma. Información Tecnológica., 2000, vol. 11, $\mathrm{N}^{\circ}$ 5, p. 171-178.

(9) GOSNELL, T. Equations for predicting defect core size for pruned radiata pine butt logs. Forest Research Institute, $1987, \mathrm{~N}^{\circ} 131.20 \mathrm{p}$.

(10) EMMINGHAM, W, S. FITZGERALD, Prunning to enhance tree and stand value. Oregon State University
Extension Service. 1995. EN: http://oasis.orst.edu/search/aEmmingham/

(11) PALAZUELOS, S. Análisis del diámetro de cilindro defectuoso en árboles podados de Pinus radiata $\mathrm{D}$. Don en la Precordillera andina de la VIII Región. Memoria para optar al título profesional de Ingeniero Forestal. Facultad de Ciencias Agrarias y Forestales, Universidad de Chile, 1995, 62 p.

(12) KNOLL, G. Radiation detection and measurement, $2^{\circ} \mathrm{ed}$. Editorial John Wiley \& Sons, 1989, 754 p.

(13) RAMIREZ, D. Aplicación de densitometría gama en la obtención de perfiles de densidad en madera de Pino Radiata (Pinus radiata D. Don). Memoria para optar al título profesional de Ingeniero Forestal. Facultad de Ciencias Agrarias y Forestales, Universidad de Chile, 1995, 73 p.

Recibido: 10.11 .04

Aceptado: 29.03.05. 